Journal of

Accident and Emergency

Medicine 1994

11, 198-200

\title{
Acute calcific periarthritis outside the shoulder: a frequently misdiagnosed condition
}

\author{
G.S.JOHNSON \& H.R. GULY \\ Accident and Emergency Department, Derriford Hospital, Plymouth PL6 8DH
}

\section{SUMMARY}

Thirteen patients presented to an accident and emergency (A\&E) department with acute calcific periarthritis of joints other than the shoulder. In only three patients was the correct diagnosis made on the initial attendance with inappropriate treatment and delay in recovery as a result. The specific features and guidelines for management of this condition are reviewed.

Key words: acute calcific periarthritis, misdiagnosis

\section{INTRODUCTION}

Patients often present to A\&E departments with acute pain related to joints but without history of injury. The differential diagnosis of these cases includes infective and inflammatory processes related to the joint and its associated structures.

Acute calcific periarthritis is a well recognized cause of shoulder pain and specific treatment may lead to rapid relief of symptoms. Less commonly, a similar process can produce symptoms in a variety of other joints.

Acute calcific periarthritis of the shoulder was first reported by Painter ${ }^{1}$ in 1907 , and Sandstrom ${ }^{2}$ in 1938 reported 320 cases of peritendinitis calcarea most of which were in the shoulder but examples in the elbow, wrist, fingers, hip, knees and toes were also noted. He divided them clinically into acute, chronic and latent forms. The acute form, which corresponds to the condition now known as acute

Correspondence: G.S. Johnson, Senior Registrar in Accident and Emergency Medicine, Derriford Hospital, Plymouth PL6 8DH, UK calcific periarthritis, was characterized by pain, local swelling, tenderness and loss of function with soft tissue calcification. A few patients had a low grade pyrexia and a raised erythrocyte sedimentation rate.

This is a self-limiting condition which usually resolves spontaneously within 4 weeks. ${ }^{3}$ Recognition of the existence of this condition outside the shoulder is poor, however, if diagnosed and appropriately treated a more rapid resolution can be achieved.

\section{CASE HISTORY}

A 44-year-old labourer was referred to the A\&E department by his general practitioner (GP) with a 4-day history of severe pain in the lateral aspect of his right knee and inability to weight bear. There was no history of trauma. The GP had made a provisional diagnosis of fibular collateral ligament injury.

On examination he was found to be mildly pyrexial $(37.8 \mathrm{C})$. There was an exquisitely tender area over the head of the fibula with erythema and reduced knee movements but no joint effusion. A radiograph was initially interpreted as showing an accessory ossicle lateral to the knee joint (Fig. 1). A diagnosis of soft tissue infection was made and he was admitted for rest, elevation and intravenous antibiotics. He was also prescribed diclofenac $50 \mathrm{mg}$ tds.

The patient's symptoms resolved rapidly over the next 2 days and prior to discharge the radiographs were interpreted correctly as showing acute soft tissue calcification consistent with acute calcific periarthritis. As this was resolving he was given no specific treatment. No bacterial growth was demonstrated from blood culture or local soft tissue washings.

\section{METHODS}

A retrospective review was made of the case notes of patients with acute calcific pariarthritis outside the shoulder seen by one of the authors (HRG) in a 4-year period. Particular note was taken of other diagnoses and inappropriate treatment administered prior to correct diagnosis and treatment. 
Acute calcific periarthritis

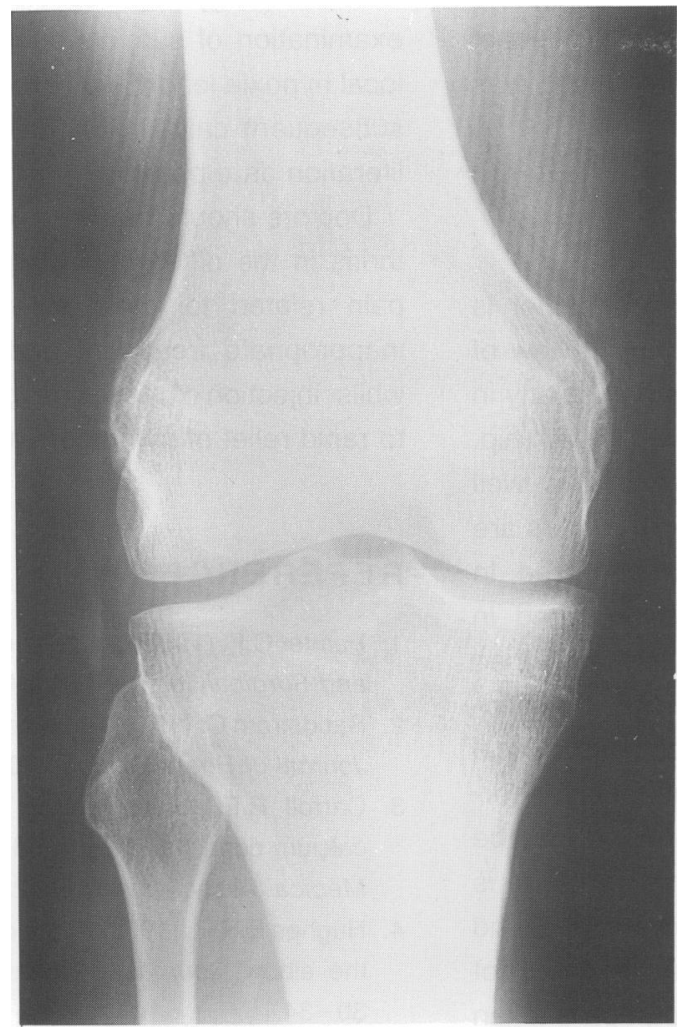

Fig. 1. Radiograph showing soft tissue calcification lateral to the joint line.

\section{RESULTS}

A total of 13 patients with acute calcific periarthritis of joints other than the shoulder were identified. The group consisted of seven males and six females with an mean age of 42.5 years (range 25 to 56 years). The sites of the disease are shown in Table 1.

The correct diagnosis was made at the initial A\&E attendance in only three patients. The diagnoses made by the GP and the medical staff in the A\&E department prior to the establishment of the correct diagnosis are shown in Table 2. The types of treatment initiated on the basis of these diagnoses included the inappropriate use of antibiotics in five cases. Use of splintage and non-steroidal antiinflammatory drugs in five cases each were appropriate despite being based on an incorrect diagnosis.

Pain was often intense and unrelieved by conventional analgesics leading to the patient reattending the department. The patients in this group had an average of one unscheduled re-attendance each prior to the correct diagnosis being made. In five patients an erroneous diagnosis of soft tissue infection was made and in two of these the infection was thought to be severe enough to require intravenous antibiotics. Local tenderness and swelling were present in all five and erythema was present in three. Two patients had a low grade pyrexia.

Once the correct diagnosis had been established, seven patients were treated by injection of locally acting steroids and local anaesthetic. This generally produced virtually immediate and complete relief of

Table 2. Incorrect diagnoses made prior to establishment of correct diagnosis

\begin{tabular}{lc}
\hline Diagnosis & No. of patients \\
\hline General practitioner diagnosis & 1 \\
Septic arthritis & 1 \\
Fibular collateral ligament injury & 1 \\
Tenosynovitis & 1 \\
Scaphoid fracture & 1 \\
Lateral epicondylitis & \\
Accident and emergency diagnoses & \\
Soft tissue infection & 5 \\
Tenosynovitis & 3 \\
Avulsion fracture & 2 \\
Neuropraxia ulnar nerve & 1 \\
Gout & 1 \\
\hline
\end{tabular}

\begin{tabular}{llll}
\hline Area & No. of cases & \multicolumn{1}{c}{ Site } & No. of patients \\
\hline Wrist & 7 & $\begin{array}{l}\text { Dorsum carpus } \\
\text { Volar carpus }\end{array}$ & 3 \\
& & Anatomical snuff box & 2 \\
Hand & 3 & Hypothenar eminence & 2 \\
& & Proximal interphalangeal jt & 1 \\
Elbow & 2 & Metacarpophalangeal jt & 1 \\
& & Common flexor origin & 1 \\
Knee & 1 & Common extensor origin & 1 \\
& & Biceps femoris & 1 \\
\end{tabular}

Table 1. Sites of acute calcific periarthritis 
G.S. Johnson \& H.R. Guly symptoms. The remaining patients were thought to be undergoing spontaneous resolution at the time of diagnosis and no further specific treatment was thought to be indicated.

\section{DISCUSSION}

Acute calcific periarthritis outside the shoulder is uncommon but not a rare condition and review of the literature reveals reports of cases particularly in the hand ${ }^{3}$ but also around the elbow, ${ }^{4}$ knee, ${ }^{5}$ hip, ankle and foot. $^{6}$ Despite the fact that it is well described, it is a condition which many doctors are unaware of and so is frequently not recognized. In this series misdiagnosis as infection occured in nearly half the patients as has been well described by others. ${ }^{2,3}$ Outside the shoulder joint, the periarticular structures are more superficial and clinical signs such as erythema, swelling and local tenderness will therefore be more prominent and may be suggestive of an infective cause. This condition is underdiagnosed as doctors are unaware of it and patients without a history of trauma often do not have radiographs taken. When a radiograph is taken the appearances of soft tissue calcification may be missed or misinterpreted as accessory bones or avulsion fractures.

Methods of treatment used include local surgical excision $^{2,4}$ and local anaesthetic injection. ${ }^{3,7}$ Results from local anaesthetic injection are rapid and long lasting pain relief with a shortened clinical course although the mechanism of this is uncertain. ${ }^{3}$ The addition of locally acting steroid is of uncertain benefit. ${ }^{8}$ Non-steroidal anti-inflammatory drugs have been used with reported success. ${ }^{7}$
The aetiology of this condition is uncertain although examination of surgical specimens has suggested local hypoxia leading to fibrocartilage formation and subsequent calcium deposition and vascular proliferation as a possible mechanism. ${ }^{9}$

Doctors should be aware of acute calcific periarthritis in the differential diagnosis of acute severe pain related to joints as misdiagnosis leads to inappropriate treatment and a delay in recovery, whilst injection of the lesion in the early stages leads to rapid relief of symptoms and return of function.

\section{REFERENCES}

1. Painter C.F. (1907) Subdeltoid bursitis. Boston Medical and Surgical Journal 156, 345-349.

2. Sandstrom C. (1938) Peritendinitis Calcarea. American Journal of Roentgenology 40, 1-21.

3. Carroll R.E., Sinton W. \& Garcia A. (1955) Acute calcium deposits in the hand. Journal of the American Medical Association 159, 422-426.

4. Hughes E.S.R. (1950) Acute deposition of calcium near the elbow. Journal of Bone and Joint Surgery 32B, 30-34.

5. Lamb D.W. (1952) Deposition of calcium salts in the medial ligament of the knee. Journal of Bone and Joint Surgery 34B, 233-235.

6. Gondos B. (1957) Observations on periarthritis calcarea. American Journal of Roentgenology 77, 93-108.

7. Greene T.L. \& Louis D.S. (1980) Calcifying tendinitis in the hand. Annals of Emergency Medicine 9, 438-440.

8. Trail I.A. (1985) Acute calcification in the fingers. Journal of Hand Surgery 10B, 263-266.

9. Uhthoff H.K., Kinti S. \& Maynard J.A. (1976) Calcifying tendinitis: a new concept of its pathogenesis. Clinical Orthopaedics 118, 164-168. 